\title{
Amyotrophic Lateral Sclerosis, a Possible Sequela of Chronic Myeloid Leukemia
}

\author{
Irsa Munir MD, Talha Mehmood MD, Isabel M. McFarlane* \\ Department of Internal Medicine, State University of New York, Downstate Medical Center, Brooklyn, N.Y, U.S.A-11203 \\ *Corresponding author: Isabel.McFarlane@downstate.edu
}

Received May 14, 2019; Revised July 26, 2019; Accepted July 31, 2019

\begin{abstract}
Amyotrophic lateral sclerosis is a fatal neurodegenerative disorder with progressive deterioration of both upper and lower motor neuron functions. It is a rare disease with one study demonstrating a prevalence of 3.9 cases per 100,000 in the USA in the year 2010-2011. It is a fatal disease with most of the deaths resulting from respiratory failure. There is no cure of this illness with some evidence supporting an improved median survival by two to three months with Riluzole (one of the agents used for treatment). Not much is known about the possible etiologies of ALS, a few studies have shown a possible likely association of ALS with various malignancies. Here we present an interesting case of a 35-year-old female with a diagnosis of chronic myeloid leukemia for seven years presented with a sub-acute decline in her motor function.
\end{abstract}

Keywords: amyotrophic lateral sclerosis, chronic myeloid leukemia, Riluzole

Cite This Article: Irsa Munir MD, Talha Mehmood MD, and Isabel M. McFarlane, "Amyotrophic Lateral Sclerosis, a Possible Sequela of Chronic Myeloid Leukemia." American Journal of Medical Case Reports, vol. 7, no. 10 (2019): 230-235. doi: 10.12691/ajmcr-7-10-3.

\section{Introduction}

Amyotrophic lateral sclerosis is a fatal neurodegenerative disorder with progressive deterioration of both upper and lower motor neuron functions. It is a rare disease with one study demonstrating a prevalence of 3.9 cases per 100,000 in the USA in the year 2010-2011 with most of the deaths resulting from respiratory failure $[1,2]$. No cure has been established with some studies showing an improved survival by a few months with Riluzole [3]. The cases of ALS are more commonly reported in the seventh to an eighth decade with one meta-analysis showing a progressive increase in the incidence of cases in the forties with maximum reported cases in sixties and seventies [4]. The etiology is unclear with current evidence showing ALS to be a multifactorial disease with both genetic and environmental factors responsible for the multi-pathway etiology of this disease [5]. A few genes have been shown to have a strong association with familial cases of ALS [5]. It is interesting that some studies have also demonstrated an increase in the incidence of ALS cases in certain malignancies with one study showing ALS could be a possible paraneoplastic symptom [6]. It is noteworthy that these studies, on one hand, highlighted the fact that while some cancers are associated with increased ALS cancer death risk like melanoma there are some other cancers like prostate cancer which may be related to reduced risks of ALS death [7]. We are presenting an interesting case of a 35-year-old female with chronic myeloid leukemia developed ALS seven years after an initial diagnosis of her malignancy.

\section{Case Presentation}

A 35-year-old female with a diagnosis of chronic myeloid leukemia for seven years presented with a worsening weakness to the emergency department. The patient was diagnosed with CML in 2010 when she presented with fatigue, weakness, and headache. At that time, her complete blood count was significant for $\mathrm{Hb}$ of $5.9 \mathrm{~g} / \mathrm{dl}$ with 400,000 WBC and massive splenomegaly. Bone Marrow was hypercellular with entire marrow replaced by myeloid lineage cells in various stages of maturation and less than 5\% blasts with RT-PCR and peripheral FISH with $80 \% \mathrm{t}(9,22)$ translocation (BCR-ABL). She was in remission on imatinib mesilate however, she stopped taking the medicines during her pregnancy in 2014. QPCR showed recurrence of the disease and imatinib mesilate was restarted. The patient was found to have a loss of molecular response on imatinib mesilate in 2017 and she was switched to nilotinib. Around the same time, the patient started complaining of weakness with difficulty climbing stairs. This weakness started before the patient was switched to nilotinib and progressed. Patient first started complaining of spasms and stiffness in multiple muscle groups followed by lower extremity weakness more pronounced on the left side than the right. Patient's weakness continued to worsen, and she presented to the emergency department after a fall. At that time, the weakness involved her bilateral lower extremities and left upper extremity with loss of hand grip. No data supports such 
presentation of motor neuron weakness secondary to tyrosine kinase inhibitors which have a reported side effect of peripheral neuropathy. Physical exam was significant for signs of both upper and lower motor neuron disorder. An extensive workup was done as her presentation warranted a broad differential. Her complete blood count and comprehensive metabolic panel showed no abnormalities. Her serum CK level was 193U/L (24-195), CRP of $9.32 \mathrm{mg} / \mathrm{dl}$ (1.0-4.0), Vitamin B 12 levels were $382 \mathrm{pg} / \mathrm{ml}$, methylmalonic acid level of 172 $\mathrm{nmol} / \mathrm{L}$ Vitamin B1 of $90.3 \mathrm{nmol} / \mathrm{L}$, serum copper level of $163 \mathrm{ug} / \mathrm{dl}$ (72-166) and zinc $70 \mathrm{mcg} / \mathrm{dl}$ (60-130). Her serum protein electrophoresis showed a normal pattern with no monoclonal bands identified on immunofixation. Multiple Cerebrospinal fluid studies were found to be normal. Her initial CSF exam showed clear, colorless CSF fluid with normal opening pressure, normal CSF cell count, CSF protein of $20 \mathrm{mg} / \mathrm{dl}$ and CSF glucose was 69 $\mathrm{mg} / \mathrm{dl}$. CSF cytology was negative. CSF ACE level was $<5$ U/L with multiple repeat CSF studies with similar findings. CSF flow cytometry for myeloid and lymphoid disorders was negative. CSF immunofixation showed no monoclonal bands. CSF viral panel for West Nile/HSV/enterovirus/HIV/CMV/EBV and VZV was negative. Serum HTLV EIA screen with western blot was found to be negative as well. The patient was also tested for multiple different autoantibodies. Her serum acetylcholine receptor binding Ab was 0.0nmol/L, MuSK antibody levels of $0.0 \mathrm{nmol} / \mathrm{L}$, negative cryoglobulins,

undetected serum levels of NMO Antibodies. MRI of the cervical, thoracic and lumbar spine performed using the following sequences: sagittal STIR, T1 weighted and T2-weighted images; axial T1-weighted and T2-weighted images for possible concern of extramedullary disease involvement but found to be an unremarkable study. Brain MRI showed nonspecific foci of T2/FLAIR signal hyperintensity in the subcortical white matter involving the left frontal lobe, bilateral parietal lobe and left basal ganglia. CT scan chest, Abdomen, and pelvis with IV contrast was essentially normal. The patient was treated multiple times with plasmapheresis and IVIG but did not improve her symptoms. The plan was then made to proceed with a sural nerve biopsy but patient went into pulseless ventricular tachycardia immediately after getting anesthesia. Later, nerve conduction studies and electromyography results were significant for electrophysiological evidence of diffuse pathology of motor neurons and their axons. After an extensive workup to rule out various pathologies and no substantial evidence, her constellation of symptoms was considered secondary to atypical presentation of ALS. Patient's overall functional status deteriorated over the course of the next few months with worsening respiratory status with eventual tracheostomy and discharge to a skilled nursing facility.

\section{Initial labs on Presentation}

Table 1. Complete Blood Count

\begin{tabular}{|c|c|c|}
\hline Serum & Patient & Reference Range \\
\hline White blood cells & 8.60 & $4.5-10.9$ \\
\hline Red blood cells & 4.15 & $4.20-5.20$ \\
\hline Hemoglobin & 13.4 & $12.0-16.0$ \\
\hline Hematocrit & 39.2 & $37.0-47.0$ \\
\hline Mean corpuscular volume $\quad(\mathrm{fL})$ & 94.6 & $78.0-95.0$ \\
\hline Mean corpuscular hemoglobin $\quad(\mathrm{pg})$ & 32.3 & $26.6-31.6$ \\
\hline MCHC* & 34.1 & $30.5-35.5$ \\
\hline Red blood cells distribution width (\%) & 14.0 & $11.5-15.1$ \\
\hline Platelets & 658 & $130-400$ \\
\hline
\end{tabular}

*mean corpuscular hemoglobin concentration.

Table 2. Comprehensive Metabolic Panel

\begin{tabular}{|c|c|c|}
\hline Serum & Patient & Reference Range \\
\hline$(\mathrm{mmol} / \mathrm{L})$ & 139 & $136-146$ \\
\hline$(\mathrm{mmol} / \mathrm{L})$ & 4.5 & $3.5-5.0$ \\
\hline$(\mathrm{mmol} / \mathrm{L})$ & 101 & $98-106$ \\
\hline Bicarbonate & 23 & $24-31$ \\
\hline Anion Gap & 15 & $5-15$ \\
\hline Blood urea nitrogen $\quad(\mathrm{mg} / \mathrm{dl})$ & 12 & $6-20$ \\
\hline Creatinine & 0.53 & $0.4-1.2$ \\
\hline BUN/Cr ratio & 22.6 & $8-23$ \\
\hline Glucose & 91 & $70-99$ \\
\hline Calcium & 9.52 & $8.4-10.3$ \\
\hline Total protein & 7.8 & $6.0-8.5$ \\
\hline Albumin & 3.9 & $2.8-5.7$ \\
\hline AST* & 20 & $10-35$ \\
\hline ALT* & 20 & $0-31$ \\
\hline ALP* & 101 & $25-125$ \\
\hline Total bilirubin & 0.62 & $0.0-1.2$ \\
\hline
\end{tabular}

*AST: aspartate aminotransferase, ALT: alanine aminotransferase, ALP: alkaline phosphatase. 
Table 3. Cerebrospinal fluid study

\begin{tabular}{|c|c|c|}
\hline Cerebrospinal fluid & Patient & Reference Range \\
\hline Appearance & Clear, colorless & Clear, colorless \\
\hline Xanthochromia & None & None \\
\hline Red blood cells (cu mm) & 12 & $<=5$ \\
\hline White blood cells (cu mm) & None seen & $0-5$ \\
\hline Protein (mg/dl) & 20 & $15-45$ \\
\hline Glucose (mg/dl) & 69 & $40-70$ \\
\hline Cytology & Negative & Negative \\
\hline Viral Culture (HSV*,VZV*, CMV*, enterovirus) & Negative & \\
\hline Angiotensin converting enzyme (U/L) & $<5$ & $<16$ \\
\hline $\mathrm{CSF}_{\mathrm{EBV}} * \mathrm{PCR}$ & Negative & Negative \\
\hline CSF West Nile PCR & Negative & Negative \\
\hline CSF HSV* $1 / 2$ PCR & Negative & Negative \\
\hline
\end{tabular}

*HSV: Herpes Simplex Virus, VZV: Varicella Zoster Virus, CMV: Cytomegalovirus, EBV: Epstein-Barr Virus.

Table 4. Reference labs: (infectious and immunological workup)

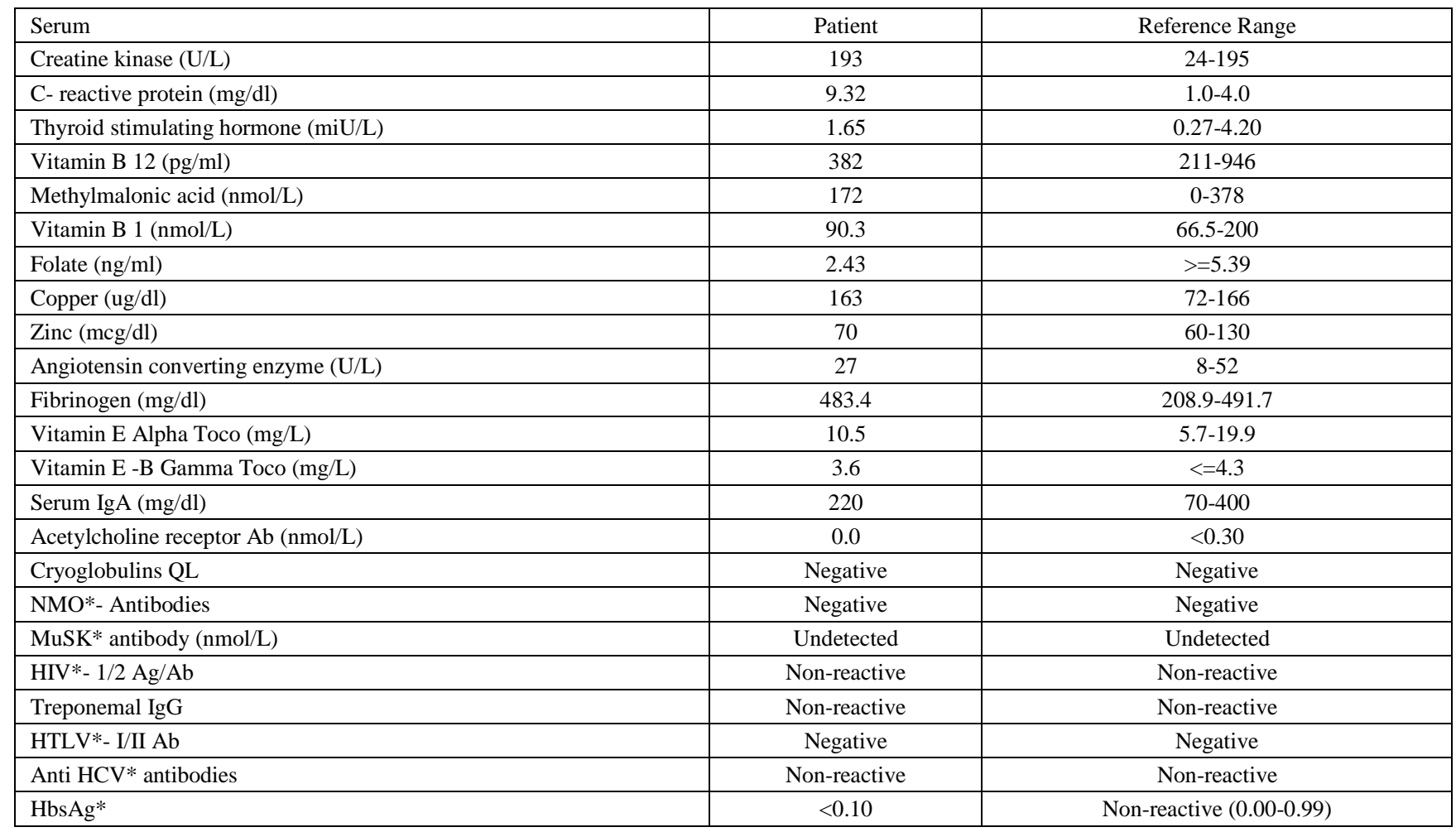

*NMO: Neuromyelitis optica, MuSK: Muscle-Specific kinase, HIV: Human Immunodeficiency Virus, HTLV: Human T-cell lymphoma Virus, HCV: Hepatitis C, HbsAg: Hepatitis B surface antigen.

Table 5. Serum protein electrophoresis

\begin{tabular}{|c|c|c|}
\hline Protein/Immunoglobulin & Patient & Reference Range \\
\hline Alpha-1-Globulin (g/dl) & 0.4 & $0.1-0.4$ \\
\hline Alpha-2-Globulin (g/dl) & 0.9 & $0.5-1.0$ \\
\hline Beta Globulin $\quad(\mathrm{g} / \mathrm{dl})$ & 1.0 & $0.5-1.0$ \\
\hline Gamma Globulin (g/dl) & 1.8 & $0.6-1.6$ \\
\hline Protein, Total $\quad$ (g/dl) & 7.6 & $6.0-8.3$ \\
\hline Albumin SPE $\quad(\mathrm{g} / \mathrm{dl})$ & 3.5 & $3.6-5.5$ \\
\hline Albumin/Globulin SPE (ratio) & 0.9 & \\
\hline IgA $\quad(\mathrm{mg} / \mathrm{dl})$ & 263 & $68-378$ \\
\hline IgG $\quad(\mathrm{mg} / \mathrm{dl})$ & 1740 & 694-1618 \\
\hline IgM $\quad(\mathrm{mg} / \mathrm{dl})$ & 175 & $40-230$ \\
\hline Immunofixation & No monoclonal bands seen & \\
\hline Kappa Light Chain (mg/dl) & 2.55 & $0.33-1.94$ \\
\hline Lambda Light Chain (mg/dl) & 1.56 & $0.57-2.63$ \\
\hline Kappa/lambda ratio & 1.63 & $0.26-1.65$ \\
\hline
\end{tabular}




\section{Imaging}

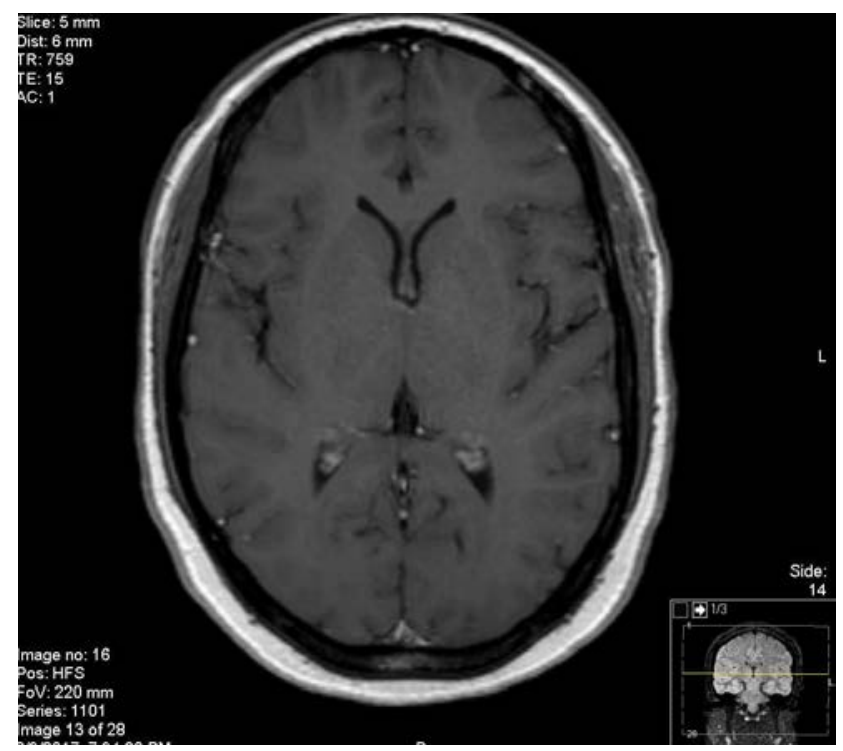

Figure 1. Brain MRI with and without contrast

Foci of T2/FLAIR signal hyperintensity in the subcortical white matter involving the left frontal lobe, bilateral parietal lobes and left basal ganglia is nonspecific and may represent inflammatory/infectious etiologies, demyelinating disease, or vasculitis.

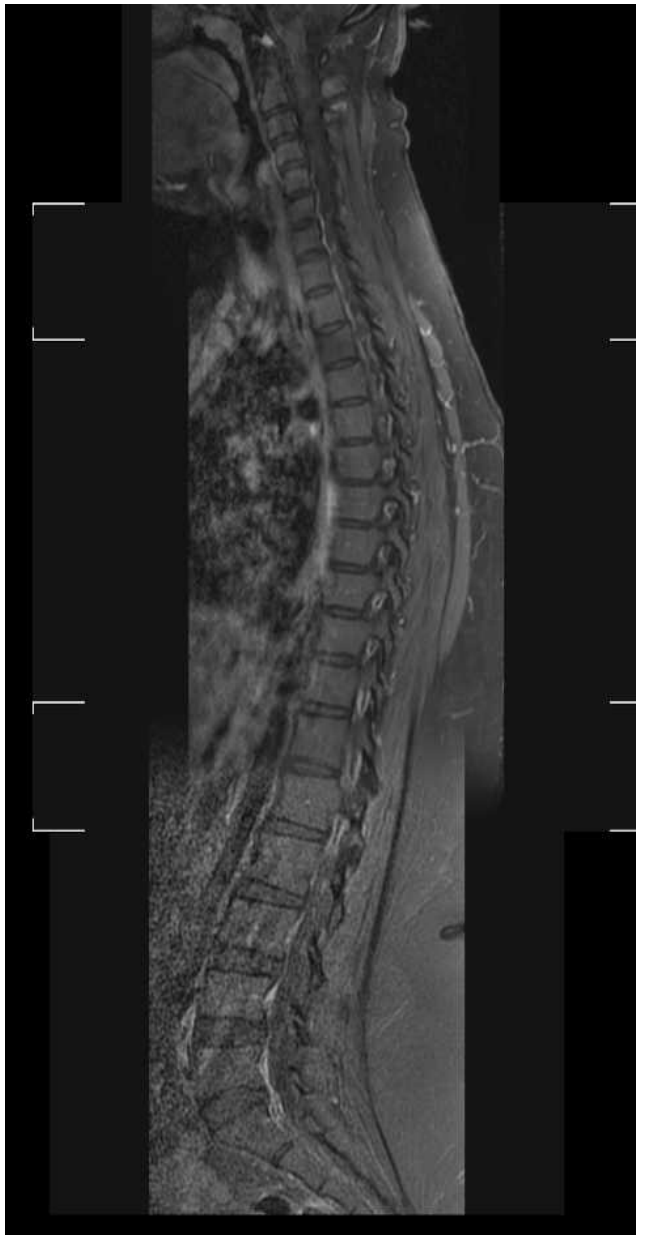

Figure 2. Spine MRI with contrast

Unremarkable MRI of the cervical, thoracic and lumbar spine. Specifically, there is no evidence of disc herniation or significant spinal canal or neural foraminal narrowing at any level. No abnormal enhancement.

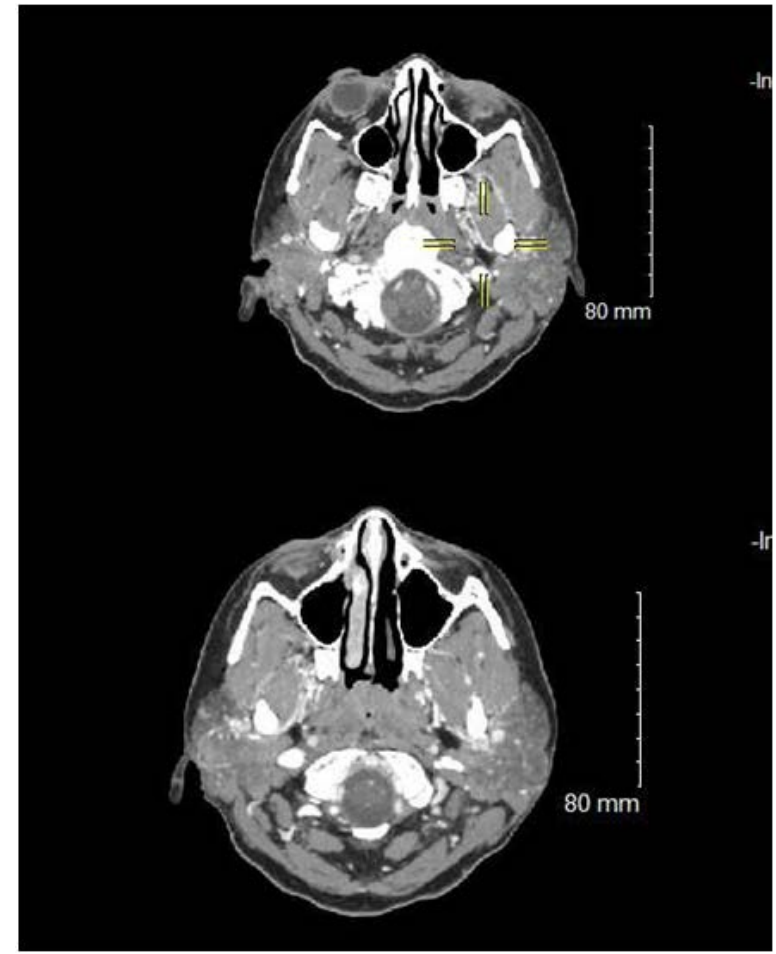

Figure 3. CT Angiography of intracranial arteries

CT Angiogram of the intracranial arteries reveals no saccular aneurysm, significant stenosis or vascular malformation.

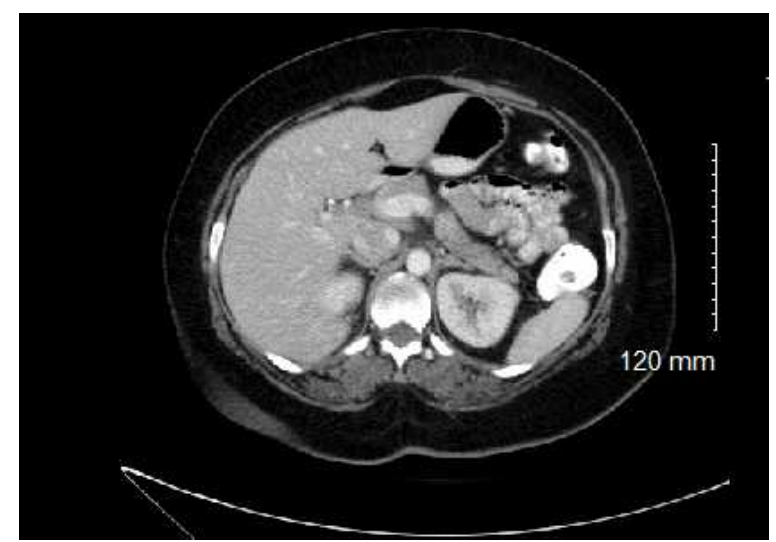

Figure 4. CT Scan Abdomen and pelvis with IV and oral contrast

The liver is mildly enlarged, measuring $19 \mathrm{~cm}$ in length indicating mild hepatomegaly, a non-specific finding. No evidence of enlarged retroperitoneal, mesenteric, pelvic lymphadenopathy or evidence of intra-abdominal neoplasm.

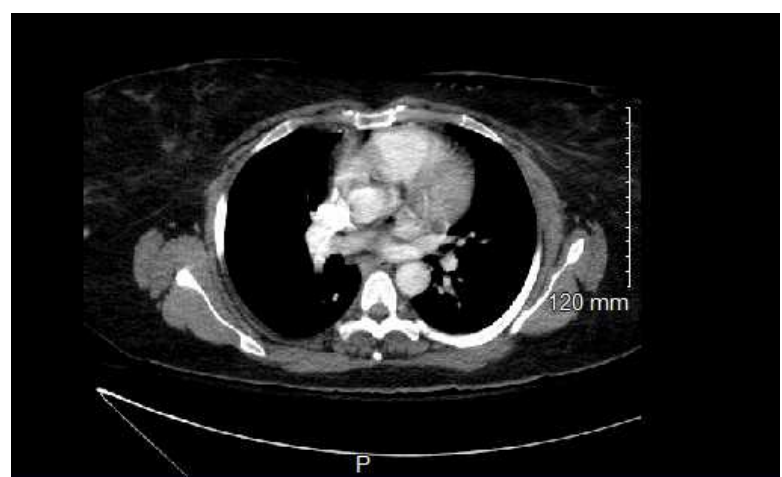

Figure 5. CT chest with IV contrast

No mediastinal or hilar lymphadenopathy. No suspicious lung mass or nodule. Unremarkable CT examination of the chest. 
Table 6. Nerve Conduction Study and Electromyography

- There is electrophysiological evidence consistent with diffuse pathology of motor neurons and their axons

- Low amplitude Right median, Left tibial, and peroneal motor evoked responses, with borderline slow Left tibial and peroneal motor conduction velocities.

- Preserved median, ulnar, left sural and superficial peroneal sensory nerve action potential (SNAP)

- The presence of active denervation in the left upper limb (deltoid), left lower limb (tibialis anterior), mid-thoracic paraspinal musculature, as well as cervical paraspinal musculature and right tongue. Active denervation was not observed in the left sternocleidomastoid. In short, active denervation was observed in 4 classic El-Escorial segments. Fasciculations were not observed.

- There are upper motor neuron findings in one segment (L knee-Joint with cross-adduction), and there is atrophy in two segments (Bilateral thenarhypothenar, and soles of both feet)

\section{Discussion}

ALS is a debilitating and progressive neurodegenerative disorder with the involvement of both upper and lower motor neurons leading to muscle weakness, functional deterioration, and death. Median survival is three to five years although there are some reported exceptions (Stephan Hawking). Etiology is deemed multifactorial with both genetic and environmental factors thought to be playing a role [5]. The incidence rates for ALS in Europe and North America range between 1.5 and 2.7 per 100,000 person-years while prevalence rates range between 2.7 and 7.4 per 100,000 person-years $[8,9,10,11]$. In the Unites States, rates of ALS are higher among the non-Hispanic white population compared with non-Hispanic blacks, Hispanics, and other racial/ethnic groups [11,12]. The incidence increases with each decade with most cases reported in the seventh to the eighth decade of life [4]. There are very few epidemiological studies and case reports explaining the possible association of ALS with various malignancies with one study suggesting ALS to be a paraneoplastic syndrome $[6,7,13,14]$. In one study, A total of 1216 ALS deaths were reported among 1-year survivors of cancer over 16.6 million person-years of follow-up [7]. Interestingly, there is an increase in ALS related death with certain malignancies (tongue, melanoma) $[7,14]$ while reduced ALS deaths risk among prostate cancer survivors [7]. There has been a study showing ALS to be a possible paraneoplastic syndrome based on the association as evidenced by increased onco-neuronal antibodies like anti-Nu, Anti-Ri and Anti-ho and co-occurrence of ALS and a neoplasm known to cause Paraneoplastic syndrome like lymphoma and breast CA [6]. It is unclear what could be the possible link between ALS and certain cancers, whether its involvement of some common genetic pathways or the side effects of treatment therapy for certain cancers. As the above-mentioned study also suggested the role of metabotropic glutamate factors in linking certain cancers like melanoma and ALS $[15,16]$. It is noteworthy as well that riluzole the commonly used drug for ALS also works on glutamate receptors and glutamate receptors subunits are also expressed in various melanomas $[15,16,17]$. Interestingly, however, a common etiologic factor, if there is any, is unclear in case of tongue cancers. In our patient, there could be a possible etiologic link between chronic myeloid leukemia and ALS unknown at this point. Initially there was a thought of possible tyrosine kinase inhibitor toxicity however TKI neuropathy is typically sensory in character.

Also, the above-mentioned study suggests that certain cancers might have a common etiologic association with ALS or certain therapies for cancer could be predisposing factors for the development of ALS. There is certainly a need to do more prospective and retrospective trials to explore the possibility of association with certain studies carried out at molecular levels to better understand the pathophysiology and commonality of genetic pathways. It can also lead to better therapeutic options to slow down the progression of ALS or to prevent further progression.

\section{Acknowledgements}

This work is supported in part by Dr. Moro O. Salifu's efforts through NIH Grant \# S21MD012474.

\section{References}

[1] Paul Mehta, M. D. "Prevalence of amyotrophic lateral sclerosis-United States, 2012-2013." MMWR. Surveillance Summaries. 65 (2016).

[2] Wolf, J., et al. "Causes of death in amyotrophic lateral sclerosis: Results from the Rhineland-Palatinate ALS registry." Der Nervenarzt 88.8 (2017): 911-918.

[3] Miller, Robert G., J. Douglas Mitchell, and Dan H. Moore. "Riluzole for amyotrophic lateral sclerosis (ALS)/motor neuron disease (MND)." Cochrane database of systematic reviews 3 (2012).

[4] Marin, Benoît, et al. "Age-specific ALS incidence: a dose-response meta-analysis." European journal of epidemiology33 (2018): 621634.

[5] Wang, Ming-Dong, et al. "Identification of risk factors associated with onset and progression of amyotrophic lateral sclerosis using systematic review and meta-analysis." Neurotoxicology 61 (2017): 101-130.

[6] Corcia, Philippe, Paul H. Gordon, and Jean-Philippe Camdessanche. "Is there a paraneoplastic ALS?." Amyotrophic Lateral Sclerosis and Frontotemporal Degeneration 16.3-4 (2015): 252-257.

[7] Freedman, D. Michal, et al. "The association between cancer and amyotrophic lateral sclerosis." Cancer Causes \& Control. 24.1 (2013): 55-60.

[8] Worms, Paul M. "The epidemiology of motor neuron diseases: a review of recent studies." Journal of the neurological sciences 191.1-2 (2001): 3-9.

[9] Logroscino, Giancarlo, et al. "Incidence of amyotrophic lateral sclerosis in Europe." Journal of Neurology, Neurosurgery \& Psychiatry 81.4 (2010): 385-390.

[10] Jordan, Heather, et al. "Amyotrophic lateral sclerosis surveillance in Baltimore and Philadelphia." Muscle \& nerve. 51.6 (2015): 815-821.

[11] Mehta, Paul, et al. "Prevalence of amyotrophic lateral sclerosisUnited States, 2014." Morbidity and Mortality Weekly Report. 67.7 (2018): 216.

[12] Roberts, Andrea L., et al. "Race/ethnicity, socioeconomic status, and ALS mortality in the United States." Neurology87.22 (2016): 2300-2308.

[13] Freedman, D. Michal, et al. "Amyotrophic lateral sclerosis mortality in 1.9 million US cancer survivors." Neuroepidemiology 25.4 (2005): 176-180. 
[14] Baade, P. D., L. Fritschi, and D. M. Freedman. "Mortality due to amyotrophic lateral sclerosis and Parkinson's disease among melanoma patients." Neuroepidemiology 28.1 (2007): 16-20.

[15] Habib, Ali Aamer, and Hiroshi Mitsumoto. "Emerging drugs for amyotrophic lateral sclerosis." Expert opinion on emerging drugs 16.3 (2011): 537-558.
[16] Stepulak, Andrzej, et al. "Expression of glutamate receptor subunits in human cancers." Histochemistry and cell biology. 132.4 (2009): 435-445.

[17] Lee, Hwa Jin, et al. "Glutamatergic pathway targeting in melanoma: single-agent and combinatorial therapies." Clinical Cancer Research 17.22 (2011): 7080-7092.

(C) The Author(s) 2019. This article is an open access article distributed under the terms and conditions of the Creative Commons Attribution (CC BY) license (http://creativecommons.org/licenses/by/4.0/). 